\title{
Evaluation of ddRADseq for reduced representation metagenome sequencing
}

\author{
Michael Y Liu $^{1}{ }^{\text {， Paul Worden }}{ }^{1}$ ， Leigh G Monahan ${ }^{1}$ ， Matthew Z DeMaere ${ }^{1}$, Catherine M Burke ${ }^{1}$, Steven P \\ Djordjevic $^{1}$, Ian G Charles ${ }^{1}$, Aaron E Darling ${ }^{\text {Corresp. } 1}$ \\ ${ }^{1}$ The ithree institute, University of Technology Sydney, Sydney, New South Wales, Australia \\ Corresponding Author: Aaron E Darling \\ Email address: aaron.darling@uts.edu.au
}

Background. Profiling of microbial communities via metagenomic shotgun sequencing has enabled researches to gain unprecedented insight into microbial community structure and the functional roles of community members. This study describes a method and basic analysis for a metagenomic adaptation of the double digest restriction site associated DNA sequencing (ddRADseq) protocol for reduced representation metagenome profiling.

Methods. This technique takes advantage of the sequence specificity of restriction endonucleases to construct an Illumina-compatible sequencing library containing DNA fragments that are between a pair of restriction sites located within close proximity. This results in a reduced sequencing library with coverage breadth that can be tuned by size selection. We assessed the performance of the metagenomic ddRADseq approach by applying the full method to human stool samples and generating sequence data.

Results. The ddRADseq data yields a similar estimate of community taxonomic profile as obtained from shotgun metagenome sequencing of the same human stool samples. No obvious bias with respect to genomic $\mathrm{G}+\mathrm{C}$ content and the estimated relative species abundance was detected.

Discussion. Although ddRADseq does introduce some bias in taxonomic representation, the bias is likely to be small relative to DNA extraction bias. ddRADseq appears feasible and could have value as a tool for metagenome-wide association studies. 
1 TITLE: Evaluation of ddRADseq for reduced representation metagenome sequencing

2

3 Michael Y. Liu ${ }^{1}$, Paul Worden ${ }^{1}$, Leigh G. Monahan ${ }^{1}$, Matthew Z. DeMaere ${ }^{1}$, Catherine M.

4 Burke $^{1}$, Steven P. Djordjevic ${ }^{1}$, Ian G. Charles ${ }^{1}$, Aaron E. Darling ${ }^{1 *}$

5

$6 \quad{ }^{1}$ The ithree institute, University of Technology Sydney, Sydney, NSW, Australia

7

8

$9 \quad$ *Corresponding Author: Aaron Darling

10 Email address: Aaron.Darling@uts.edu.au

11

12

13

14

15

16

17

18

19

20

21

22

23

24 


\section{Abstract}

27 Background. Profiling of microbial communities via metagenomic shotgun sequencing has 28 enabled researches to gain unprecedented insight into microbial community structure and the 29 functional roles of community members. This study describes a method and basic analysis for a 30 metagenomic adaptation of the double digest restriction site associated DNA sequencing 31 (ddRADseq) protocol for reduced representation metagenome profiling.

32 Methods. This technique takes advantage of the sequence specificity of restriction 33 endonucleases to construct an Illumina-compatible sequencing library containing DNA 34 fragments that are between a pair of restriction sites located within close proximity. This results 35 in a reduced sequencing library with coverage breadth that can be tuned by size selection. We 36 assessed the performance of the metagenomic ddRADseq approach by applying the full method 37 to human stool samples and generating sequence data.

38 Results. The ddRADseq data yields a similar estimate of community taxonomic profile as 39 obtained from shotgun metagenome sequencing of the same human stool samples. No obvious 40 bias with respect to genomic $\mathrm{G}+\mathrm{C}$ content and the estimated relative species abundance was 41 detected.

42 Discussion. Although ddRADseq does introduce some bias in taxonomic representation, the bias 43 is likely to be small relative to DNA extraction bias. ddRADseq appears feasible and could have 44 value as a tool for metagenome-wide association studies. 


\section{Introduction}

49 'Who is doing what' is the ultimate open question in microbiome studies. Shotgun metagenomics

50 is often applied to gain knowledge of functional roles for bacteria in microbial communities,

51 where the data can be used to predict protein encoding genes and enzymatic pathways present in

52 the community, sometimes leading to testable hypotheses for microbial function. Advances in

53 DNA sequencing technology and computing have dramatically accelerated the development of

54 sequence-based metagenomics, which has been proposed by many scientists as a means to

55 characterize the function of microbes in microbiomes (Handelsman 2004; Simon \& Daniel

56 2011). Nevertheless, studies seeking to link microbial community phenotype to genes in the

57 metagenome, e.g. metagenome-wide association studies (MGWAS) can require large numbers of

58 samples to be processed. As a result, MGWAS on genetically diverse samples such as

59 mammalian faeces and soil remain difficult or intractable, due to the prohibitive cost of shotgun

60 metagenome sequencing to adequate depth.

61

62 Here, we investigate the potential of reduced representation sequencing to be used for low-cost 63 metagenome profiling. We describe a metagenomic adaptation of ddRADseq (Peterson et al.

64 2012), a method for genotyping by sequencing studies of large and complex individual genomes

65 (e.g. plants). This approach takes advantage of the sequence specificity of restriction

66 endonucleases, where the genomic DNA is first fragmented by restriction digestion to construct a

67 set of sequences that are flanked by the targeted restriction sites. This results in a sequencing

68 library with complexity that is reduced roughly in proportion to the density of the restriction

69 enzyme cut-sites, with a complexity that is tunable via fragment size selection. The design of this

70 approach also includes a dual-index combinatorial barcoding approach allowing samples to be 
71 multiplexed in a single sequencing run. We demonstrate the method on human stool samples and

72 compare the recovered taxonomic profiles to those obtained by standard metagenome shotgun

73 sequencing on the same samples.

74

75 Materials \& Methods

76 Ethical approval for this study was obtained from the University of Technology Sydney Human

77 Research Ethics Committee (Approval number: UTS HREC REF NO. 2014000448).

78

79 Three healthy adult stool samples were collected and the DNA extracted using an UltraClean Microbial DNA isolation kit (MO BIO Laboratories). The ddRADseq metagenome libraries were generated following the original ddRADseq protocol (Peterson et al. 2012), which uses a double restriction digest followed by size selection to construct a library containing only a defined subset of the total genomic DNA, with fine-scale control of library complexity available via size selection. Combinations of commercially available restriction enzymes were computationally evaluated for cut site density and other properties in a set of reference genomes chosen to reflect

86 a wide range of $\mathrm{G}+\mathrm{C}$ content. The evaluation suggested a short list of optimal enzyme 87 combinations, and the combination of NlaIII and HpyCH4IV (New England BioLabs) was 88 selected for their properties of buffer compatibility, insensitivity to dam methylation, overhang 89 incompatibility, and heat sensitivity. This combination of restriction enzymes was predicted to 90 minimise bias in the representation of individual species within the metagenomic community. 91 The protocol employed a dual index approach for sample barcoding where each adapter carries a 92 sample barcode compatible with standard Illumina multiplexing index reads (Figure 1). Finally, a 93 variable length region containing randomly synthesised nucleotides is included immediately 
94 downstream of the read priming site, to improve cluster calling and offer the potential to identify

95 PCR duplicates via unique molecular identifiers (Kivioja et al. 2011). The full list of adapters

96 designed and used in this study is outlined in the Supplementary Information.

97

98 To generate the ddRADseq libraries, 50ng of DNA from each sample was used in a restriction 99 double digest following the reaction conditions recommended by the enzyme manufacturer. A 100 total of $5 \mathrm{U}$ of both enzymes were used in the reaction and subsequently heat inactivated 101 following the manufacturer's instructions. A generous molar ratio of 1:40 (digested DNA: 102 sequencing adapters) was then used for sequencing adapter ligation, which ensured an excess of 103 adapters for ligation. Amplification of the adapter-ligated fragments was then carried out using 104 the Illumina standard P5 and P7 flowcell oligo primers. The three sequencing libraries were then 105 pooled with equal volume and size selected for approximately 500-600 bp fragments with 106 SPRIselect beads (Beckman Coulter) using a $0.5 \times / 0.6 \times$ double-sided clean-up. Shotgun 107 metagenomic libraries were prepared from separate aliquots of sample DNA using the Illumina 108 Nextera DNA kit. Sequencing of those samples were done with half a lane of the Illumina HiSeq 1092500 in rapid PE250 mode. Details of the library construction protocol are given in the 110 Supplementary Information.

Quality assurance of sequencing data and availability of data

113 The quality of sequence data was examined using FastQC (Andrews 2010). Reads were also 114 checked for the presence of the sequenced portion of the restriction enzyme recognition site 115 (NlaIII: CATG, HpyCH4IV: CGT) starting at position 0-3 in the read depending on the phasing 116 of the barcode adapter associated with each sample. Shotgun sequence data are available from 
117 the NCBI Short Read Archive, project accession SRP100899, containing two datasets per 118 sample.

\section{Results}

121 We present a full method for sample preparation using the double digest restriction site

122 associated DNA sequencing (ddRADseq) technique. Paired-end Illumina sequencing was carried 123 out on both shotgun and ddRADseq libraries for three human gut microbiome samples. As a pilot 124 study with only three samples, there is insufficient statistical power to evaluate associations 125 between phenotype and microbiome genotype in these samples. Instead, we evaluate the extent 126 to which ddRADseq yields a similar estimate of community taxonomic profile as that obtained 127 from shotgun metagenome sequencing of the same samples. If the ddRADseq is strongly biased 128 against (or in favour of) some taxonomic groups it might reduce (or increase) power to detect 129 associations between phenotype and the protein coding genes in the genomes of that group. Both 130 the shotgun and ddRADseq reads were taxonomically profiled using MetaPhlAn (Segata et al. 131 2012), which counts reads matching to clade-specific protein-coding marker genes in order to 132 estimate taxon abundance from metagenomic read data. The taxonomic profile between 133 individuals was different on both the genus and species levels, consistent with the inter134 individual variation that is typically observed in human microbiome studies. Highly similar, but 135 not identical, taxonomic profiles were observed between the shotgun-sequenced and ddRADseq 136 metagenome libraries. Hierarchical clustering using the Manhattan distance showed that 137 ddRADseq samples cluster closely with their matching shotgun samples (Figure 2). These results 138 suggest that the representation of protein coding genes in ddRADseq metagenomes may be 139 uniform enough to support association studies. 
141 To assess whether the restriction enzyme pair used in this study created a reduced representation

142 library that was biased by genomic $\mathrm{G}+\mathrm{C}$ content we plotted the estimated relative abundance of

143 each taxonomic group with respect to the genomic $\mathrm{G}+\mathrm{C}$ content of a reference genome from

144 that taxonomic group. As can be seen in Figure 3, if an association exists between genomic $\mathrm{G}+$

$145 \mathrm{C}$ and relative abundance in our ddRADseq libraries, it is not visually apparent. We refrain from

146 reporting Spearman correlations on this dataset, as such correlation tests have well known

147 problems on compositional data (Lovell et al. 2015).

\section{Discussion}

150 Changes in the human microbiota have been associated with complex disease. The gut 151 microbiota, for example, has been linked to conditions such as irritable bowel syndrome and 152 inflammatory bowel disease. Major research efforts are now underway to move beyond 153 taxonomic associations to discover and describe the genetic basis for these microbiome154 associated diseases. Although shotgun metagenomics is one way to carry out those analyses, it 155 can be prohibitively expensive in some situations. Therefore, reduced representation techniques 156 such as ddRADseq may provide a useful means to enable large-scale studies within a fixed 157 research budget. A metagenomic adaptation of the traditional ddRADseq protocol is feasible in 158 principle, yet no data has previously been reported for such an application to real microbiome 159 samples. Here we presented a preliminary analysis of the data and showed that metagenome 160 profiling with ddRADseq appears to be feasible. Taxon relative abundances estimated from 161 clade-specific marker genes are similar but not identical to estimates from shotgun metagenomic 162 data, implying that protein coding gene profiles from ddRADseq would likewise be similar to 
163 those obtained with shotgun metagenomics. The potential savings that could be obtained by the

164 use of ddRADseq instead of shotgun metagenomics depends on several factors, including read

165 length, community complexity, and the chosen degree of complexity reduction. To understand

166 the potential cost savings, consider a set of high complexity samples (e.g. soil) to be sequenced

167 on the highest output Illumina instruments, currently the NovaSeq 6000, which yield paired end

$168150 \mathrm{nt}$ reads on fragments about $400 \mathrm{bp}$ long. Assuming size-selection has been tuned to generate

169 ddRADseq fragments about $300 \mathrm{nt}$ long, with fragment start sites separated in the genomes by

170 about 30,000 bp on average, this would represent a complexity reduction of $99 \%$. A study which

171 aims to associate markers with some phenotypic or other sample data would then in principle

172 require 99\% less data to find an association of equivalent statistical significance. In this example,

173100 times as many samples could be processed with ddRADseq over shotgun metagenome

174 sequencing, with equal statistical power and at equal cost in sequencing (though library

175 preparation costs would increase). The trade-off is that phenotypic features are associated to

176 markers, for example the abundance of individual ddRADseq fragments or single nucleotide

177 variants within those fragments, rather than the potentially causal genotypic features themselves.

178 Nevertheless, such markers may still be highly useful as they could provide a lead for further

179 investigation of the features of genomes containing those markers. Therefore, when combined

180 with careful fragment size selection, ddRADseq profiling could have value as a cost effective

181 means to generate metagenomic profiles for use with metagenome-wide association studies and

182 as a complementary tool for surveillance of microbial ecosystems, tracking differences across 183 environments, treatments or time scale. 
185 Even if enzyme choice leads to some bias in metagenomic ddRADseq libraries, other sources of 186 bias may be more significant. For example, DNA extraction efficiency for individual community 187 members can depend heavily on features such as the cell wall architecture of those organisms, 188 leading to extreme bias in the extracted DNA and final sequencing library (Frostegard et al. 189 1999; Morgan et al. 2010). The biases introduced by ddRADseq may be small in comparison to 190 those introduced by DNA extraction. Sensitivity of restriction enzymes to methylation and other 191 poorly understood DNA modifications maybe another factor contributing towards the observed 192 differences in detection. The cleavage of target DNA may be blocked, or impaired, when a 193 particular base in the enzyme's recognition site is modified. Future work to test this could 194 explore the use of enzymes that have the same recognition site (isoschizomers), but with 195 different methylation sensitivity, to identify modified bases through changes in the efficiency of 196 restriction digest. Metagenomic ddRADseq will also be affected by biases that are commonly 197 seen in single organism RADseq experiments including polymorphism in restriction site, 198 mutation leading to novel restriction sites and heterogeneity in base composition across or 199 between genomes (Andrews et al. 2014; Andrews \& Luikart 2014; Arnold et al. 2013; DaCosta 200 \& Sorenson 2014; Lowry et al. 2017; McCluskey \& Postlethwait 2015; Puritz et al. 2014).

201

\section{Conclusions}

203 This study presents a proof of concept for adopting double digest restriction site associated DNA 204 sequencing (ddRADseq) against microbiome dataset. The generated sequencing results and 205 analysis demonstrate: (1) the feasibility of adopting the ddRADseq profiling technique on 206 complex microbiome samples; (2) the ability of this new technique to generate a reduced 207 representation of a complex microbial community, and with taxon relative abundances that are 
208 similar to the community profile from shotgun metagenome sequencing of the same samples; (3)

209 the reduced representation generated by the enzymes used in the ddRADseq method does not

210 exhibit an obvious bias with respect to $\mathrm{G}+\mathrm{C}$ content of genomes in the sample, which will

211 support the wider application of such technique against other environmentally relevant samples.

212 The method and data presented here may be of value to the research community, because the 213 technique promises to enable metagenome-wide association studies in systems where it was 214 previously intractable due to scale or complexity.

215

216 Acknowledgements

217 We would like to acknowledge the Centre for Digestive Diseases in Sydney, NSW, Australia for 218 their assistance with fecal sample collection and for helpful discussions.

\section{References}

228 Andrews KR, Hohenlohe PA, Miller MR, Hand BK, Seeb JE, and Luikart G. 2014. Trade-offs 229 and utility of alternative RADseq methods: reply to Puritz et al. Mol Ecol 23:5943-5946.

$230 \quad 10.1111 /$ mec.12964 
231 Andrews KR, and Luikart G. 2014. Recent novel approaches for population genomics data 232 analysis. Mol Ecol 23:1661-1667. 10.1111/mec.12686

233 Andrews S. 2010. FastQC: a quality control tool for high throughput sequence data. Available 234 online at: http://wwwbioinformaticsbabrahamacuk/projects/fastqc.

235 Arnold B, Corbett-Detig RB, Hartl D, and Bomblies K. 2013. RADseq underestimates diversity 236 and introduces genealogical biases due to nonrandom haplotype sampling. Mol Ecol 22:3179237 3190. 10.1111/mec.12276

238 DaCosta JM, and Sorenson MD. 2014. Amplification biases and consistent recovery of loci in a 239 double-digest RAD-seq protocol. PLoS One 9:e106713. 10.1371/journal.pone.0106713

240 Frostegard A, Courtois S, Ramisse V, Clerc S, Bernillon D, Le Gall F, Jeannin P, Nesme X, and 241 Simonet P. 1999. Quantification of bias related to the extraction of DNA directly from soils. 242 Appl Environ Microbiol 65:5409-5420.

243 Handelsman J. 2004. Metagenomics: application of genomics to uncultured microorganisms. 244 Microbiol Mol Biol Rev 68:669-685. 10.1128/MMBR.68.4.669-685.2004

245 Kivioja T, Vaharautio A, Karlsson K, Bonke M, Enge M, Linnarsson S, and Taipale J. 2011. 246 Counting absolute numbers of molecules using unique molecular identifiers. Nat Methods 9:72247 74. 10.1038/nmeth. 1778 
248 Lovell D, Pawlowsky-Glahn V, Egozcue JJ, Marguerat S, and Bahler J. 2015. Proportionality: a

249 valid alternative to correlation for relative data. PLoS Comput Biol 11:e1004075.

250 10.1371/journal.pcbi.1004075

251 Lowry DB, Hoban S, Kelley JL, Lotterhos KE, Reed LK, Antolin MF, and Storfer A. 2017.

252 Breaking RAD: an evaluation of the utility of restriction site-associated DNA sequencing for 253 genome scans of adaptation. Mol Ecol Resour 17:142-152. 10.1111/1755-0998.12635

254 McCluskey BM, and Postlethwait JH. 2015. Phylogeny of zebrafish, a "model species," within 255 Danio, a "model genus". Mol Biol Evol 32:635-652. 10.1093/molbev/msu325

256 Morgan JL, Darling AE, and Eisen JA. 2010. Metagenomic sequencing of an in vitro-simulated 257 microbial community. PLoS One 5:e10209. 10.1371/journal.pone.0010209

258 Peterson BK, Weber JN, Kay EH, Fisher HS, and Hoekstra HE. 2012. Double digest RADseq: 259 an inexpensive method for de novo SNP discovery and genotyping in model and non-model 260 species. PLoS One 7:e37135. 10.1371/journal.pone.0037135

261 Puritz JB, Matz MV, Toonen RJ, Weber JN, Bolnick DI, and Bird CE. 2014. Demystifying the 262 RAD fad. Mol Ecol 23:5937-5942. 10.1111/mec.12965

263 Segata N, Waldron L, Ballarini A, Narasimhan V, Jousson O, and Huttenhower C. 2012.

264 Metagenomic microbial community profiling using unique clade-specific marker genes. Nat 265 Methods 9:811-814. 10.1038/nmeth.2066 
266 Simon C, and Daniel R. 2011. Metagenomic analyses: past and future trends. Appl Environ

267 Microbiol 77:1153-1161. 10.1128/AEM.02345-10

268 
Figure 1

Diagrammatic representation of the ddRADseq method: the oligos, adapters and final sequencing library.

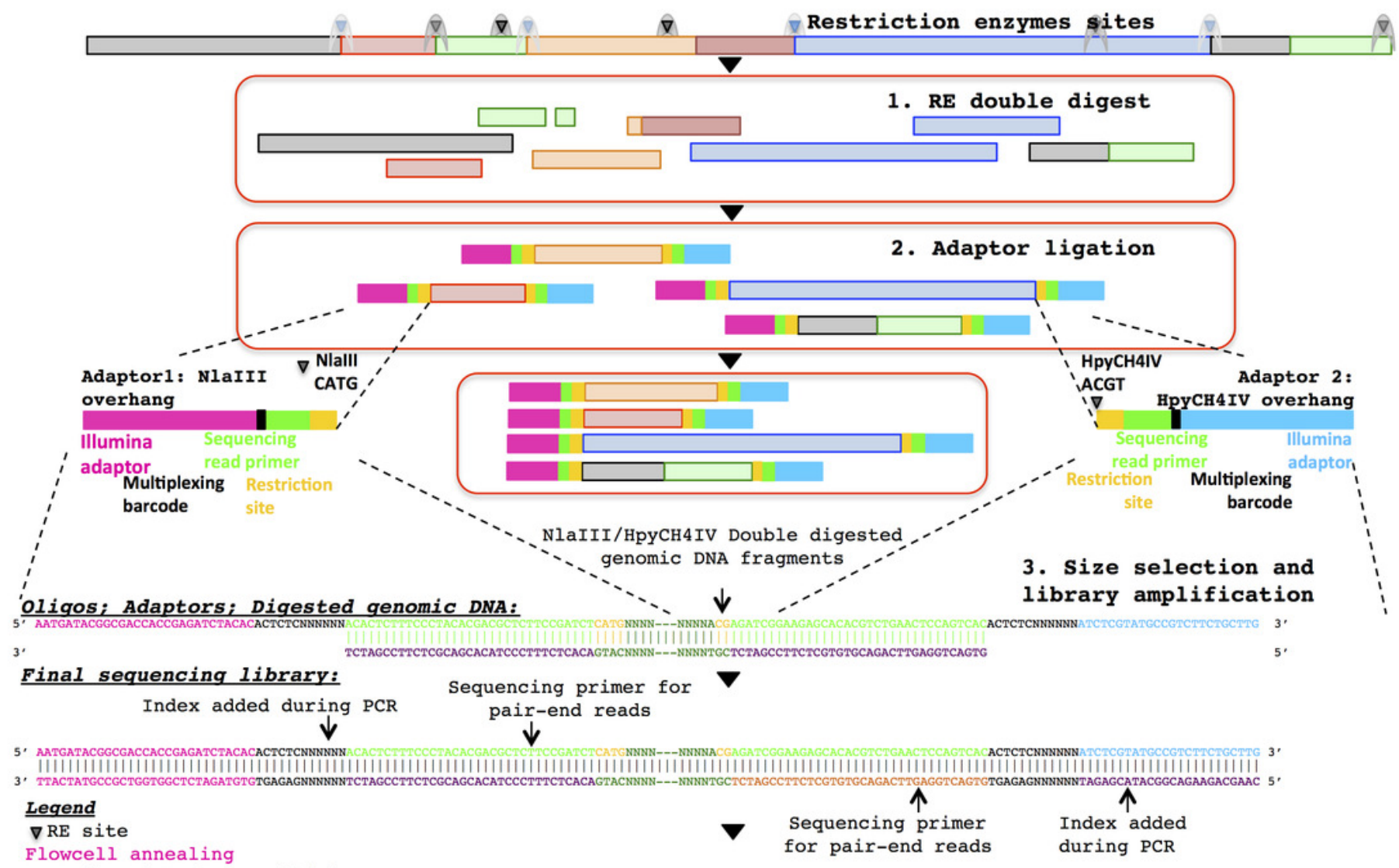

Barcode and Index - multiplex

Genomic DNA

4. Sequencing and analyze

$\begin{array}{lll}\text { READ } & 1 & \text { primer } \\ \text { READ } & 2 \text { primer }\end{array}$ 
Figure 2

Abundance of specific phylotypes detected in both shotgun and ddRADseq libraries.

A) Samples clustered at the genus level; B) Samples clustered at the species level. The heatmap is hierarchically clustered based on predicted abundances using the Manhattan distance metric.

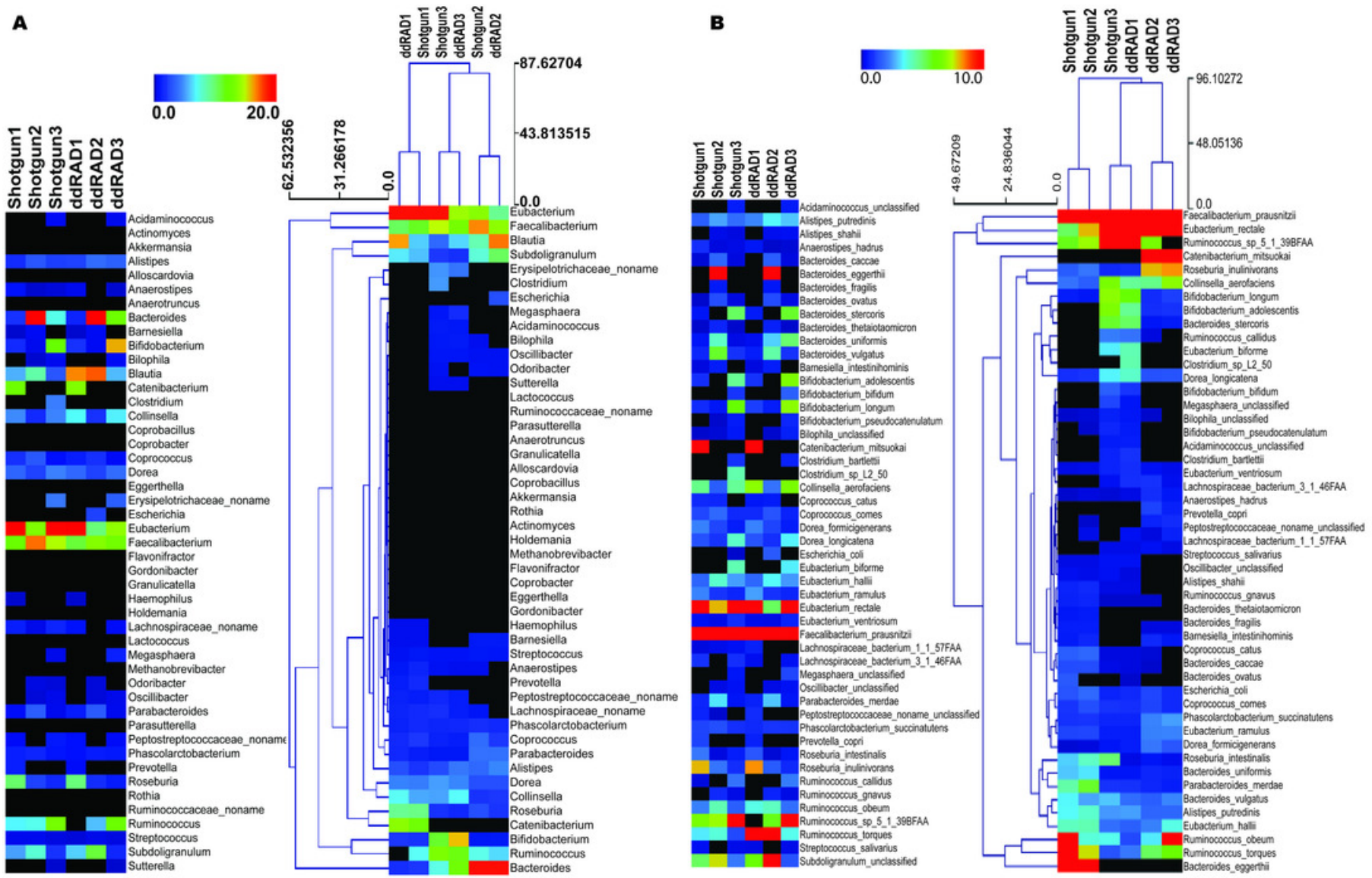


Figure 3

Abundance of taxonomic groups in both shotgun and ddRADseq libraries as a function of genomic $\mathrm{G}+\mathrm{C}$ content for all three samples.

Although differences in estimated relative abundance exist between shotgun and ddRADseq libraries, the ddRADseq protocol we used has not created a bias with an obvious association to genomic $\mathrm{G}+\mathrm{C}$ content.

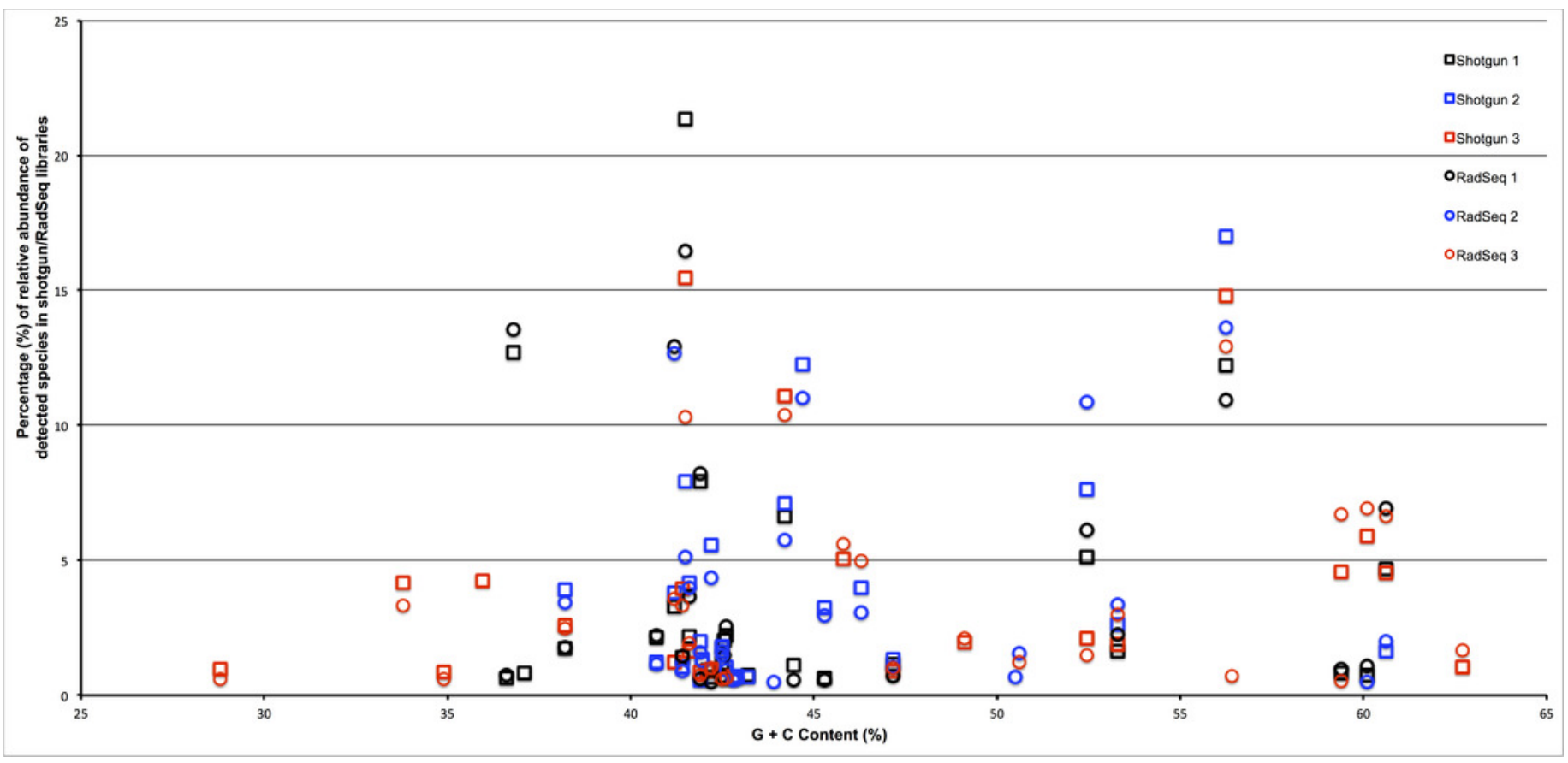

